\title{
QSOs from a Variability-and-Proper Motion Survey
}

\author{
Helmut Meusinger, Jens Brunzendorf \\ Thüringer Landessternwarte Tautenburg, D-07778 Tautenburg, Germany
}

Ralf-Dieter Scholz

Astrophysikalisches Institut Potsdam, An der Sternwarte 16, D-14482

Potsdam, Germany

Mike Irwin

Institute of Astronomy, Madingley Road, Cambridge CB3 1HA, UK

\begin{abstract}
We report on a search for QSOs via variability and zeroproper motion. Candidates were selected by means of indices for overall variability, long-term variability, proper motion and image structure measured on a large number of Schmidt plates with a time-baseline of three decades. Spectroscopic follow-up observations of the candidates brighter than the pre-estimated completeness limit, $B_{\lim } \approx 19.7$, of the survey revealed about 200 QSOs and Seyfert 1s with redshifts $z \approx 0-3$. We describe the survey strategy and discuss the properties of the resulting QSO sample.
\end{abstract}

\section{Introduction}

Most criteria for the selection of QSO candidates are based on the differences of their spectral energy distribution (SED) compared to stars. All such search methods are known to be biased (e.g., Hewett \& Foltz 1994). Alternative strategies are provided by fundamental properties of quasars that are not directly based on the SED, like variability of flux densities (e.g., Hawkins 1983; Véron \& Hawkins 1995; Bershady et al. 1998) and stationarity of positions (e.g., Kron \& Chiu 1981). The search for objects which are both variable and stationary is supposed to be a powerful technique for efficiently finding QSOs. The selection bias of such a search strategy is expected to be quite different from those of more conventional optical search techniques. A combination of both constraints was successfully applied by Majewski et al. (1991) in a small survey field. Here, we use a similar search strategy and present preliminary results from a new combined variability and proper motion QSO search (in the following "VPM survey") in two Tautenburg Schmidt fields.

For an efficient VPM quasar search, a large number of homogeneous observations of a large number of faint objects are needed, with high astrometric and photometric accuracy and covering a long time-baseline of a few decades. These requirements can be matched only by archival plates from large Schmidt 
telescopes. The basic observational material of our VPM survey is provided by a large number of plates from the Tautenburg Schmidt telescope. In several fields, the Tautenburg plate archive contains homogeneous material of more than a hundred plates with epoch differences of up to three decades or more. In addition, due to its relatively large focal length the Tautenburg Schmidt plates have fewer problems with plate bending and are well suited for astrometry. A plate covers an area of about 10 square degrees.

\section{Survey Strategy and Candidate Selection}

For the VPM survey, we selected the fields centred on the globular clusters M3 and M92, respectively, where large numbers of $\mathrm{B}$ plates are available with a time-baseline of about 30 years and with typical limiting magnitudes between about 19.5 and 21.5. Whereas the basic strategy is the same for both fields, there are several differences in the details (see Scholz et al. 1997; Brunzendorf \& Meusinger 2001). For the M3 field, which is at high Galactic latitude, $57 \mathrm{~B}$ plates were selected and measured with the APM facility in Cambridge. In the M92 field $\left(b \approx 35^{\circ}\right)$, where the search faces the problem of stronger contamination by foreground stars, a larger number of $162 \mathrm{~B}$ plates were selected and digitised by means of the Tautenburg plate scanner TPS. In both fields, several U- and Vplates were digitised as well in order to derive colour indices (which are, however, not used for the candidate selection).

The basic object sample contains all objects which are measured on a given number $(n>3)$ of plates. The analysis of the objects from these samples comprises the following steps: 1.) classification according to the image structure and selection of star-like objects, 2.) computation of relative proper motions from the linear regression of the measured positions as a function of the epochs; the goodness of fit is taken as a measure of the proper motion error, 3.) transformation of relative proper motions to absolute proper motions using several hundred faint galaxies per field as reference frame; the measured proper motion in units of the proper motion error is taken as a proper motion index, 4.) computation of the variability indices for the star-like objects, and 5.) specification of the thresholds for the selection of stationary and variable objects.

The frequency distributions of the measured mean $\mathrm{B}$ magnitudes for all star-like objects indicate that substantial incompleteness sets in at $B \approx 20$. We adopt $B \approx 19.7$ for the completeness limit of the survey in both fields. Typical proper motion errors are less than about 2 mas/yr up to $\mathrm{B}=19$ and about 4 mas/yr at the survey limit. The photometric errors, expressed by the standard deviation of the magnitude measurements of an object on all plates, are typically less than $0.1 \mathrm{mag}$ for $B<18$ and rise in the $\mathrm{M} 3$ field up to $\approx 0.2 \mathrm{mag}$ at the survey limit. In a first attempt of the data reduction in the M92 field (Brunzendorf \& Meusinger 2001), this increase was considerably stronger, obviously due to a lower accuracy of the procedures applied there for the determination of the object parameters. Meanwhile, we have repeated the photometry for the M 92 field using the SExtractor package by Bertin \& Arnouts (1996) which yields a substantial reduction of the photometric uncertainties (Brunzendorf \& Meusinger, in preparation). 

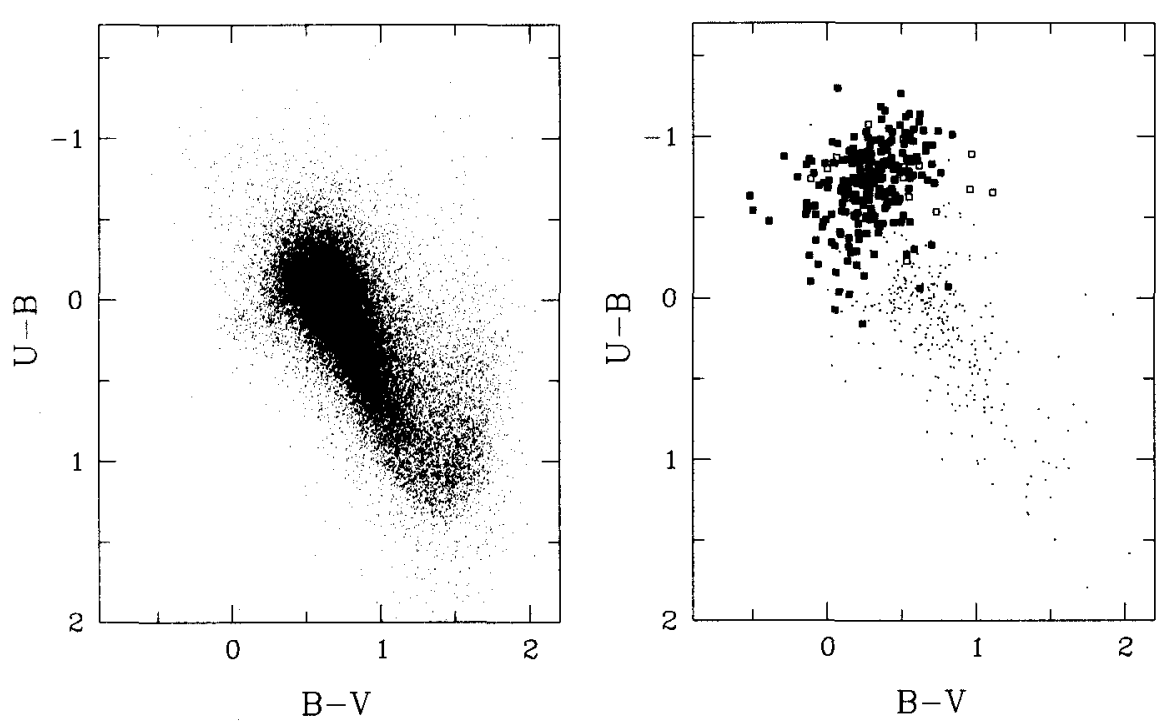

Figure 1. Colour-colour diagrams for all star-like objects in the two VPM search fields (left) and for the spectroscopically observed candidates right). On the right-hand side, QSOs are shown as filled squares, Seyfert 1s as open squares and stars as dots.

The variability of an object is assessed by measuring the deviation of the individual magnitudes about the mean magnitude, and is normalized by the average magnitude scatter for star-like objects in the same flux range. In the M3 field, which overlaps with the blue grens CFHT survey (Crampton et al. 1989), the CFHT QSOs were used to define the variability selection thresholds in such a way that a success rate of at least $40 \%$ is expected in combination with a high completeness of $90 \%$. In the M92 field, where no QSOs were known before, we had to define indices that are directly related to the probability of real variability: an object is considered a candidate if its probability for variability is larger than $95 \%$. In addition, an index for long-term variability is computed which measures variability with time-scales longer than about a few months. The long-term variability index is defined either by means of structure function analysis (Scholz et al. 1997) or by the method of mean square successive differences (Brunzendorf \& Meusinger 2001).

The basic samples contain 24,600 objects in the M 3 field and 35,000 objects in the M92 field. After the exclusion of the objects near the plate margins and in the crowded cluster region and considering only star-like objects, the basic samples are reduced by a factor of 4 in the M 3 field and 2 in the M 92 field, respectively. About $70 \%$ of the objects from these reduced basic samples are rejected due to the zero-proper motion constraint. Finally, the variability selection strongly reduces the candidate sample to a manageable size of altogether about 300 candidates of high or medium priority. 


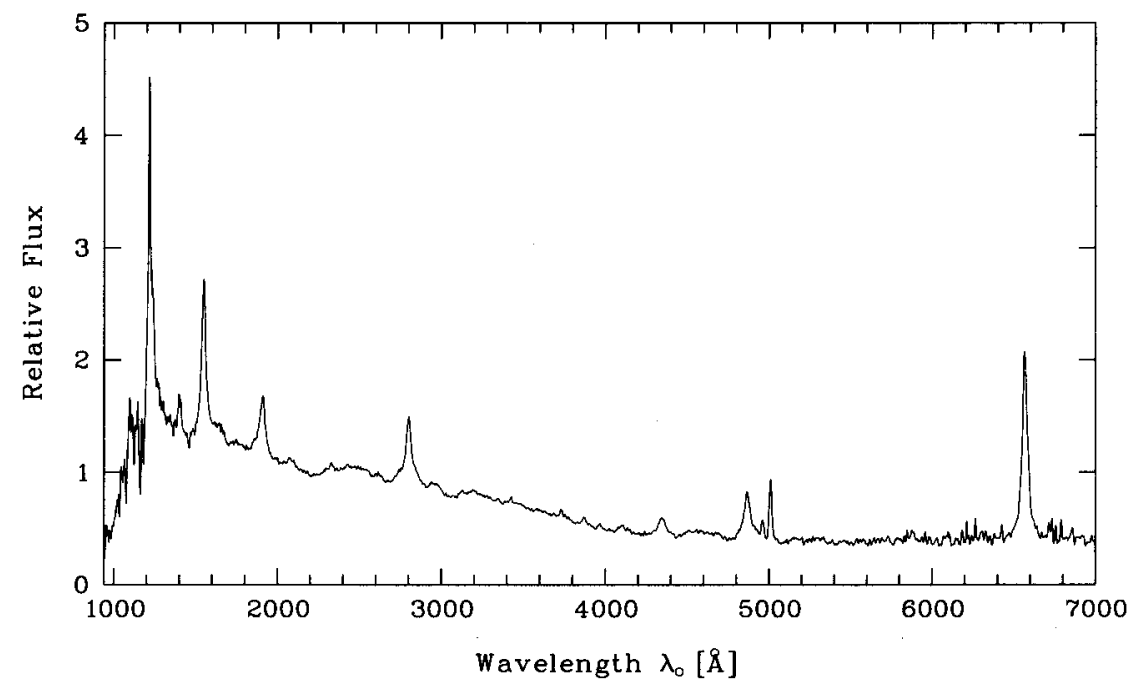

Figure 2. Composite spectrum (in the rest frame) obtained from the follow-up spectra of the QSOs and Seyfert 1s in the M92 field.

\section{Properties of the AGN Sample}

Follow-up spectroscopy was performed for the brighter candidates with the Tautenburg multi-object spectrograph TAUMOK. The fainter candidates were observed with the faint-object spectrograph CAFOS at the $2.2 \mathrm{~m}$ telescope of the $\mathrm{DSAZ}^{1}$, Calar Alto, Spain. Up to now, follow-up spectra are available and reduced for altogether 412 candidates. $182 \mathrm{QSOs}$ and Syls were found, corresponding to a total success rate of $44 \%$. Preliminary results for the M92 field were presented by Meusinger \& Brunzendorf (2001). (The publications of the results for the M 3 field and from the improved photometry in the M92 field, respectively, are in preparation.)

Despite a few BAL QSOs and a few objects with relatively weak broad emission lines, there is no indication of a substantial population of QSOs with anormalous SEDs. As can be seen from the colour-colour diagram in Fig. 1, all candidates with extremely red colours proved to be galactic foreground stars. Further, nearly all of the VPM QSOs populate the area of the colour-colour diagram which would be surveyed by a classical two-colour search. Up to the limit of the present survey, the QSO population detected by the VPM method is obviously not significantly different from the population detected by colour surveys. The same conclusion is reached from the colour-redshift diagrams and from the detailed analysis of the spectra. The composite spectrum of the VPM QSOs (Fig. 2) is very similar to those of quasars from other optical surveys.

\footnotetext{
${ }^{1}$ German-Spanish Astronomical Centre operated by the Max-Planck-Institute for Astronomy, jointly with the Spanish National Comission for Astronomy
} 


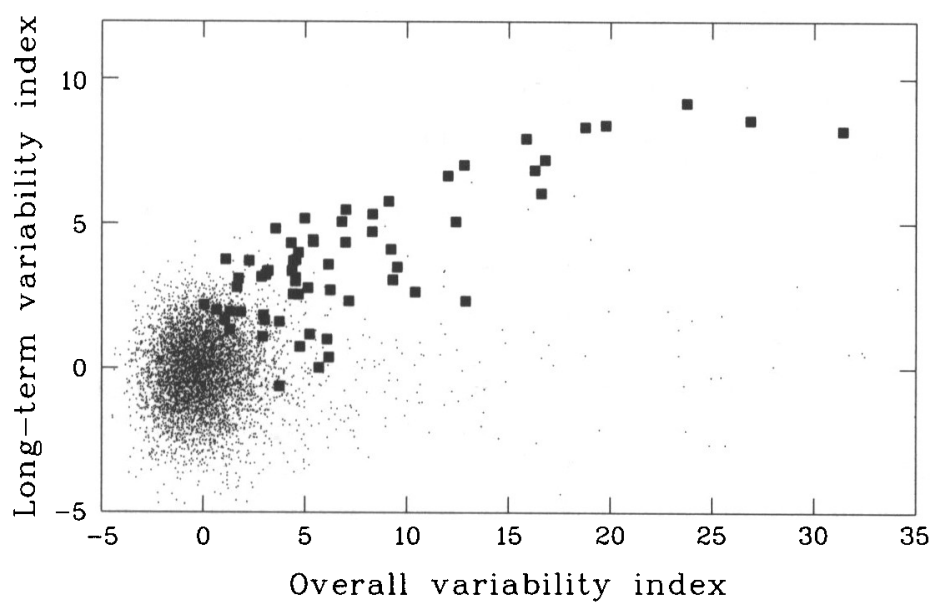

Figure 3. Long-term variability versus overall variability for the starlike objects with zero-proper motions (dots) and QSOs/Seyfert 1s (filled squares) in the M92 field.

For practical reasons, we divided the candidate sample into three priority classes. In the high priority class, all 141 candidates were observed with a success rate as high as $96 \%$ in the M3 field and $71 \%$ in the M92 field. High priority means that both overall variability and long-term variability are highly significant. In addition, spectra were taken for a number of medium-priority candidates. QSO candidates of low priority were observed especially when only one of the selection criteria was slightly violated. Figure 3 shows the long-term variability index versus the index for total variability. Evidently, quasars show in many, yet not in all, cases strong long-term variability. This confirms the importance of the long-term variability index, but also the use of both variability indices for an efficient QSO variability search.

The redshift distributions are similar to those from colour surveys. A difference appears in the preliminary results from the M 92 field, where higher redshifts are slightly underabundant. This can be understood as due to the incompleteness of the variability selection at fainter magnitudes where the photometric accuracy is lower. A (preliminary) completeness of $50 \%$ was estimated in the M 92 field by the comparison of the magnitude-dependence of the variability selection threshold with the distribution of the variability indices computed for the (more complete) QSO sample investigated by Hook et al. (1994). For the M 3 field we estimate a completeness of $90 \%$ from the overlap with the CFHT QSOs. The cumulative surface density of VPM QSOs in the two search fields is remarkably high, especially for $B \leq 18.5$ (Fig. 4).

\section{Conclusion}

The combination of constraints for overall variability, long-term variability and zero-proper motion provides an efficient (yet expensive) AGN search method 

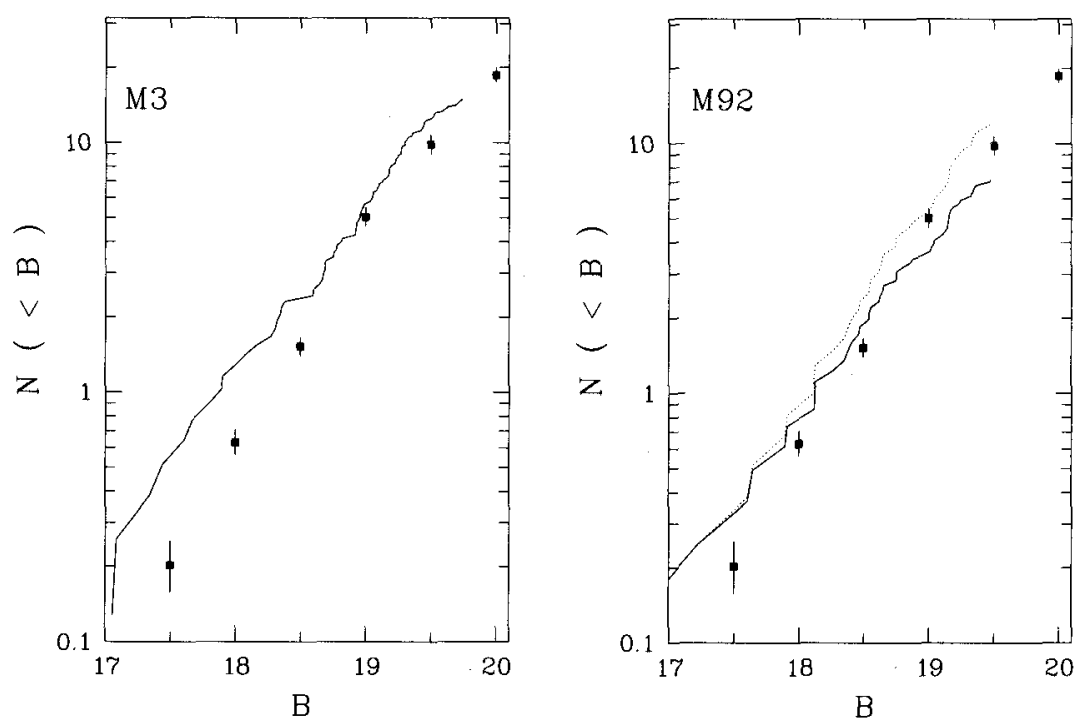

Figure 4. Cumulative surface densities of QSOs from the VPM survey (solid curves) compared with the data by Hartwick \& Schade (1990) from conventional optical surveys (squares and error bars). The dotted curve for the M92 field is the result of correction for incompleteness.

that yields sizeable quasar samples. Up to the limit of the present survey, the properties of the VPM-QSO sample do not significantly differ from those of the QSOs from more conventional optical searches. The resulting QSO sample will be well suited to investigate the long-term variability of QSOs.

\section{References}

Bershady, M.A., Trevese, D., \& Kron, R.G. 1998, ApJ, 496, 103

Bertin, E., \& Arnouts, S. 1996, A\&AS, 117, 393

Brunzendorf, J., \& Meusinger, H. 2001, A\&A, 373, 38

Crampton, D., Cowley, A.P., \& Hartwick, F. 1989, ApJ, 345, 59

Hartwick F.D.A., \& Schade, D. 1990, ARA\&A, 28, 437

Hawkins, M.R.S. 1983, MNRAS202, 571

Hewett, P.C., \& Foltz, C.B. 1994, PASP, 106, 113

Hook, I.M., McMahon, R.G., Boyle, B.J. et al. 1994, MNRAS, 268, 305

Kron, R.G., \& Chiu, L.-T. 1981, PASP, 93, 397

Majewski, S R., Munn, J.A., Kron, R.G., et al. 1991, ASP Conf. Ser. 21, 55

Meusinger, H., \& Brunzendorf, J. 2001, A\&A, 374, 878

Scholz, R.-D., Meusinger, H., \& Irwin, M. 1997, A\&A325, 457

Véron, P., \& Hawkins, M.R.S. 1995, A\&A296, 665 